> Les lésions tissulaires ou les pertes d'organes provoquent des changements structuraux et métaboliques qui peuvent être à l'origine de graves complications. L'ingénierie tissulaire (IT), dont l'objectif thérapeutique est de recréer, régénérer ou restaurer la fonction d'un tissu lésé, est la coalescence de trois éléments: un biomatériau d'origine synthétique ou biologique, dégradable ou non, des cellules réparatrices et des signaux (hypoxie, contraintes mécaniques, morphogènes, etc.). Le cartilage articulaire, l'os et les vaisseaux font partie des tissus pour lesquels I'IT s'est développée considérablement, de la recherche fondamentale jusqu'aux essais cliniques. Si les biomatériaux doivent présenter des propriétés différentes en fonction du tissu à régénérer, la composante cellulaire de I'IT est majoritairement représentée par les cellules souches, au premier rang desquelles les cellules souches mésenchymateuses adultes prélevées à partir de la moelle osseuse ou du tissu adipeux. Ces dernières années, des progrès ont été accomplis dans la compréhension des mécanismes biologiques qui régissent la différenciation des cellules souches et dans le développement de matériaux aux propriétés biologiques et physicochimiques contrôlées. Cependant, de nombreux verrous technologiques et réglementaires devront être levés avant que l'ingénierie tissulaire puisse passer du laboratoire à la clinique et entrer dans l'arsenal thérapeutique de la médecine régénératrice. Cette revue a pour objectif de souligner les progrès récents accomplis dans l'utilisation des cellules souches en ingénierie des tissus ostéoarticulaires et vasculaires. <

\section{Les cellules souches \\ en ingénierie des tissus ostéoarticulaires et vasculaires}

Claire Vinatier, Laurence Bordenave, Jérôme Guicheux, Joëlle Amédée

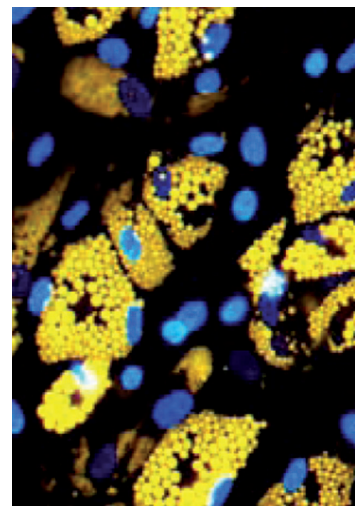

J. Guicheux : Inserm U791, LIOAD (Laboratoire d'ingénierie ostéo-articulaire et dentaire) groupe STEP, Université de Nantes, 1, place Alexis Ricordeau, 44042 Nantes, France. C Vinatier: Inserm U791, LIOAD, groupe STEP, Université de Nantes; Graftys SA,

Aix-en-Provence, France. J. Amédée : Inserm U577, Université Victor Segalen Bordeaux 2,

L'ingénierie tissulaire (IT) est une discipline dont l'objectif est de développer des substituts biologiques capables de restaurer, maintenir ou améliorer la fonction des tissus lésés. Pour cela, elle associe les principes de l'ingénierie des matériaux et des sciences de la vie (cellules réparatrices et signaux biologiques, biochimiques ou biophysiques).

146, rue Léo Saignat, 33076 Bordeaux, France. L. Bordenave : Inserm U577, Université Victor Segalen Bordeaux 2 ;

CIC-IT Biomatériaux Inserm, CHU de Bordeaux, France. joelle.amedee@inserm.fr

La médecine régénératrice des tissus squelettiques (os, cartilage) et vasculaires a récemment bénéficié des progrès de l'IT pour faire son entrée en clinique humaine. Concernant les matériaux, et en fonction de la nature du tissu à régénérer, ceux-ci doivent présenter des propriétés physicochimiques (composition, élasticité, injectabilité, etc.) différentes [1], adaptées aux propriétés biologiques, mais également les propriétés biomécaniques des tissus cibles. Les cellules réparatrices utilisées en IT sont majoritairement représentées par les cellules souches et en particulier les cellules souches mésenchymateuses (CSM) adultes prélevées à partir de différents tissus : moelle osseuse [2], sang de cordon, sang adulte, cordon ombilical, périoste, muscle, tissu adipeux [3], membrane synoviale. Cependant, au vu de la diversité des applications cliniques de l'ingénierie tissulaire, l'identification des cellules les mieux adaptées est toujours source de débat au sein de la communauté scientifique : cellules autologues ou allogéniques, cellules souches adultes ou embryonnaires, cellules de moelle osseuse ou de tissu adipeux, etc. 


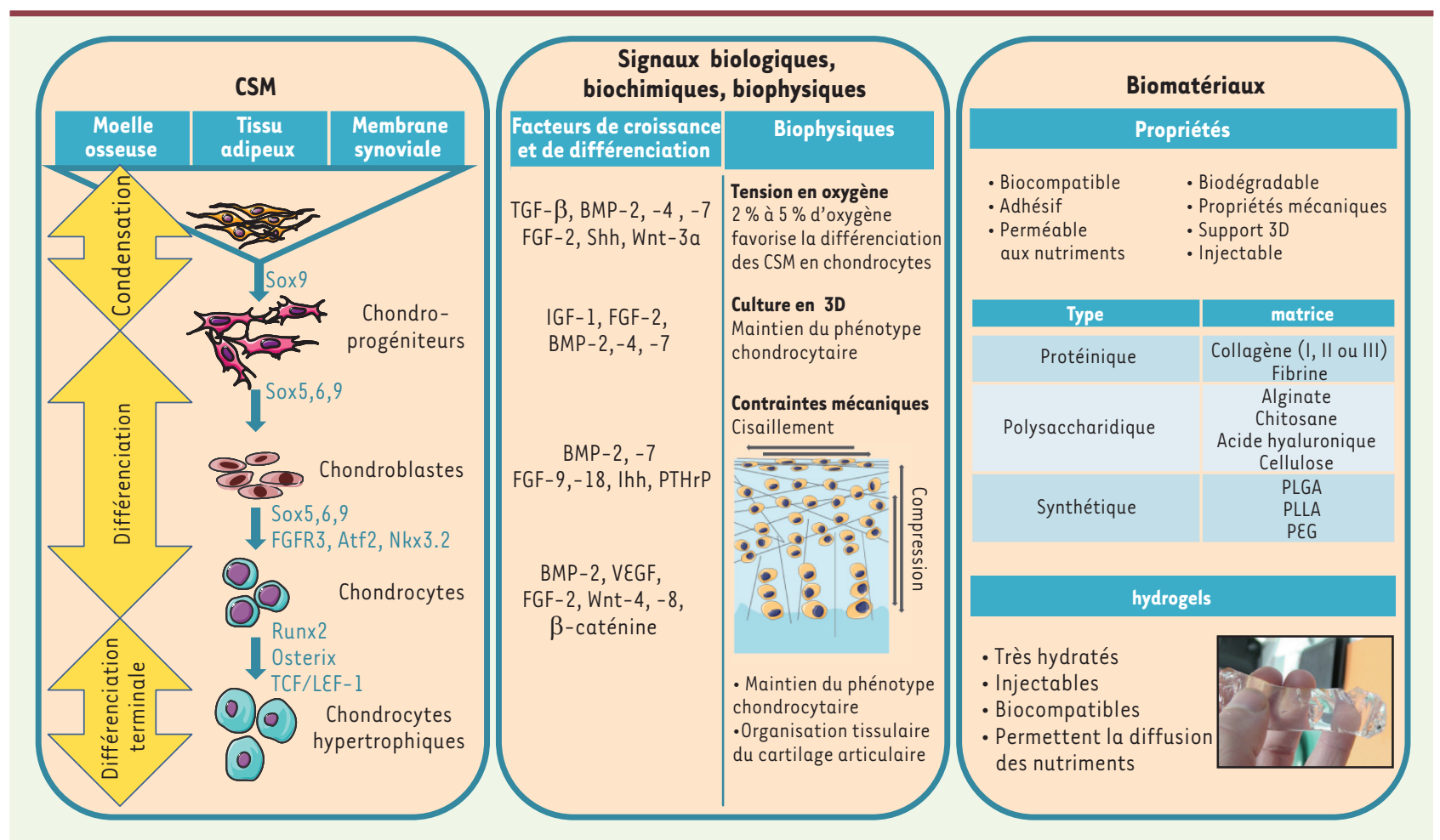

Figure 1. Ingénierie tissulaire du cartilage articulaire: cellules souches mésenchymateuses et biomatériaux. Sur le panel de gauche les étapes successives de la différenciation en cellules du cartilage à partir des différents progéniteurs de la moelle osseuse, du tissu adipeux et de la membrane synoviale sont indiquées. Sur le panel central, les molécules intervenant à chacune de ces étapes, et sur le panel de droite les différents biomatériaux analysés.

\section{Les cellules souches en ingénierie du cartilage articulaire}

Le cartilage articulaire (CA) est un tissu conjonctif hautement spécialisé situé à l'extrémité des os longs. Il est constitué de chondrocytes et de quelques cellules progénitrices. La matrice extracellulaire (MEC) du cartilage articulaire est richement hydratée et composée d'un réseau de collagènes fibrillaires (collagènes de types II, IX et XI) ainsi que de protéoglycanes dont l'aggrécane. Lorsqu'il est endommagé, le CA ne se régénère pas en raison principalement de son absence de vascularisation. II a donc été proposé d'utiliser les techniques d'ingénierie tissulaire et de thérapie cellulaire pour régénérer ce tissu. Les CSM adultes semblent être de bonnes candidates pour l'IT du cartilage, puisqu'elles sont capables de se différencier vers le lignage chondrogénique [4] (Figure 1). Parmi les différentes sources de CSM, celles qui proviennent de la membrane synoviale présentent le meilleur potentiel de différenciation chondrogénique en termes de cinétique de différenciation, de prolifération et de quantité de protéines matricielles produites in vitro, mais également en termes de réparation de lésions cartilagineuses in vivo [5]. La différenciation chondrogénique des CSM est décrite comme reproduisant le processus d'ossification endochondrale [6]. Ainsi, les facteurs TGF- $\beta 1$ (transforming growth factor) et IGF-1 (insulin growth factor), connus pour favoriser la différenciation chondrogénique au cours de l'ossification endochondrale (Figure 1) ont été utilisés avec succès pour différencier les CSM en chondrocytes [7].

Des facteurs environnementaux impliqués dans I'homéostasie articulaire influencent également la différenciation chondrogénique des CSM. Parmi ceux-ci : une faible tension en oxygène [8], les contraintes mécaniques [9] et la culture en trois dimensions ont été largement décrites. La différenciation chondrogénique des CSM, comme dans l'ossification endochondrale, ne s'arrête pas au stade du chondrocyte mature mais se poursuit vers l'hypertrophie du chondrocyte, la minéralisation de la MEC et la formation d'os [10]. Afin de prévenir le risque de formation d'un tissu minéralisé notamment in vivo, il semble cependant possible de contrôler ce processus en cocultivant les CSM avec des chondrocytes articulaires [11]. Par ailleurs, I'hypoxie, outre son action stimulatrice sur la différenciation chondrogénique précoce des CSM, pourrait également être utilisée pour prévenir l'apparition de marqueurs du stade terminal de différenciation chondrogénique [12]. Malgré le grand nombre de données in vitro conférant aux CSM des propriétés intéressantes pour la régénération du cartilage, seules de rares études cliniques ont 
été recensées. Celles-ci utilisent préférentiellement des CSM de moelle osseuse associées à des éponges de collagènes permettant, sous un lambeau périosté ou synovial, d'observer une réparation du cartilage [13-15]. Ces études concluent généralement à une supériorité de la transplantation de CSM autologues sur les techniques chirurgicales classiques (microfracture ou débridement). Globalement, les lésions cartilagineuses traitées par transplantation de CSM sont comblées par un tissu de nature cartilagineuse, améliorant de ce fait les signes cliniques, mais la nature hyaline de ce tissu cartilagineux n'a jamais été prouvée. Ces transplantations de CSM sont par ailleurs réalisées au décours d'actes chirurgicaux souvent invasifs (arthrotomie ${ }^{1}$ ). Afin de pouvoir recourir à des techniques d'implantation moins invasives (arthroscopiques), I'utilisation de biomatériaux injectables s'est rapidement imposée comme une solution cliniquement pertinente.

Aujourd'hui, les biomatériaux utilisés en IT du cartilage ont donc pour objectif, non seulement de permettre la transplantation par une voie non invasive des cellules sur le site de la lésion, mais aussi d'être des supports tridimensionnels (3D) permettant la différenciation chondrogénique des CSM [4]. Ces biomatériaux sont à base de protéines, de polysaccharides ou synthétiques (Figure 1) et ont déjà été utilisés pour la différenciation des CSM [16]. Parmi ces biomatériaux, les hydrogels ont récemment reçu une attention particulière. Ils sont composés de polymères visqueux synthétiques ou naturels qui possèdent la particularité de former des réseaux 3D après réticulation physique, ionique ou covalente. Ces hydrogels ont une teneur en eau proche de celle du cartilage, ce qui leur permet de mimer l'environnement 3D physiologique des cellules. Les hydrogels présentent également l'avantage d'être injectables et peuvent donc être implantés, après mélange avec les cellules, par chirurgie arthroscopique mini-invasive.

Finalement, parmi les nombreux concepts d'IT du cartilage articulaire en développement, seuls quelques-uns dépasseront le stade des études précliniques. Malgré les récents progrès enregistrés tant en biologie des CSM qu'en physico-chimie des biomatériaux, de nombreux paramètres doivent encore être affinés. La différenciation chondrogénique des CSM doit en particulier être très finement contrôlée (prévention de I'hypertrophie, stabilité du phénotype) et la biodégradation des matériaux mieux maîtrisée. Gageons cependant que les années prochaines verront l'émergence de cette discipline, à la frontière de la biologie et de la physicochimie, ouvrir de nouvelles fenêtres thérapeutiques en médecine régénératrice, notamment du cartilage articulaire.

\section{Les cellules souches dans l'ingénierie du tissu osseux}

À la différence du cartilage, le tissu osseux est un tissu conjonctif minéralisé, vascularisé, qui a la capacité de se renouveler tout au long de la vie par un processus de remodelage assuré par deux types cellulaires : les ostéoclastes qui résorbent la matrice osseuse et les ostéoblastes qui forment une nouvelle matrice extracellulaire. Un déséqui-

${ }^{1}$ L'arthrotomie est un geste chirurgical qui consiste à ouvrir la capsule articulaire pour accéder à l'articulation. L'arthroscopie est une technique chrirurgicale mininvasive qui introduit un instrument optique de 3 ou $4 \mathrm{~mm}$ de diamètre appelé arthroscope dans une articulation par une très courte incision. libre entre la résorption et la formation osseuse conduit à des pathologies osseuses comme l'ostéoporose et a pour conséquence des modifications importantes de la masse et de l'architecture osseuses. Celles-ci peuvent être à l'origine de fractures et de pertes massives de tissu osseux, difficiles à réparer sans stratégies de régénération tissulaire. Ces stratégies doivent tenir compte de la physiologie de ce tissu (minéralisé, vascularisé et innervé) et de sa fonction mécanique.

Les techniques d'ingénierie du tissu osseux sont à ce jour très prometteuses, notamment pour la régénération des pertes massives de tissu osseux. Ces techniques associent des cellules réparatrices $[17,18]$ qui doivent s'orienter vers le lignage ostéoblastique sous l'action de différents facteurs biochimiques, matriciels et mécaniques.

De nombreuses sources cellulaires peuvent être utilisées pour des applications en ingénierie du tissu osseux (Figure 2). Compte tenu des limites des cultures primaires de cellules humaines différenciées autologues obtenues à partir d'os trabéculaire ou alvéolaire [19] (quantité limitée de cellules, faible capacité de prolifération et de différenciation), les recherches se sont orientées rapidement vers l'utilisation de sources alternatives de cellules utilisables en clinique. Les cellules embryonnaires offrent d'intéressantes caractéristiques, en particulier leur pluripotence. La littérature décrit les nombreux stimulus (addition de facteurs ostéogènes, culture en micromasse) utilisés pour engager leur différenciation vers le lignage ostéoblastique [20] mais leur application dans le domaine de l'ingénierie tissulaire soulève des problèmes réglementaires et éthiques. Les cellules souches pluripotentes induites (iPS) devraient offrir dans les années à venir un potentiel considérable pour la médecine régénératrice. Leur orientation vers le lignage ostéoblastique a été montrée récemment [21] mais de nombreux obstacles technologiques doivent être levés pour une utilisation en ingénierie tissulaire. À ce jour, l'utilisation de CSM adultes semble être encore la plus prometteuse pour des applications cliniques [2].

\section{Différenciation des CSM vers le lignage ostéogénique}

De nombreux facteurs environnementaux gouvernent la différenciation des CSM vers le lignage ostéoblastique (Figure 2) [22]. En effet, une des caractéristiques du tissu osseux est d'abriter la moelle osseuse, siège de I'hématopoïèse contenant les cellules souches hématopoïétiques (CSH) nichées dans un tissu stromal composé des CSM et de nombreuses autres cellules (fibroblastes, 


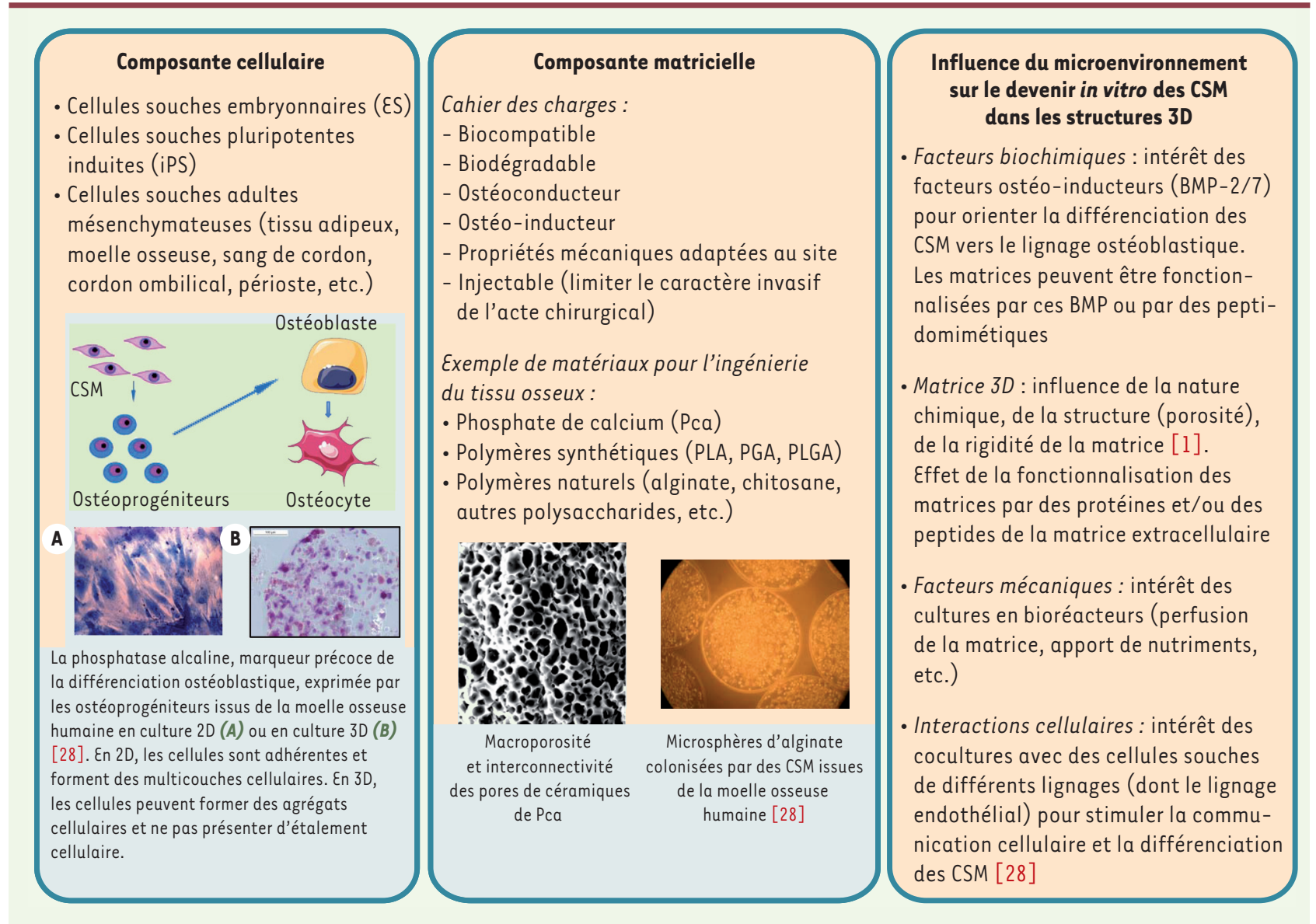

Figure 2. Ingénierie tissulaire du tissu osseux : les cellules souches mésenchymateuses et leur microenvironnement.

cellules endothéliales, adipocytes, etc.). Les activités de ces cellules hématopoiétiques et stromales sont étroitement liées par des interactions cellule/cellule ou cellule/matrice extracellulaire et par l'intermédiaire de facteurs locaux (facteurs de croissance, morphogènes et cytokines) et hormonaux. De plus, ce tissu est en permanence soumis à des sollicitations mécaniques. Ce microenvironnement cellulaire, biochimique, matriciel et mécanique dans lequel se trouvent ces CSM peut être en partie mimé in vitro dans des modèles de culture en deux et trois dimensions, par l'addition de facteurs biochimiques, par l'utilisation de bioréacteurs mimant des contraintes mécaniques, ou encore par des supports matriciels comme structures d'hébergement de ces cellules qui peuvent orienter la différenciation de ces CSM.

Dans des modèles de culture en 2D, les étapes de la différenciation ostéoblastique sont bien identifiées et sont sous le contrôle de différents facteurs de transcription (cbfal/runx2, osterix, Dlx5) [23]. Dans des structures $3 \mathrm{D}$, les mécanismes sont beaucoup moins bien connus. Ces CSM peuvent être induites in vitro vers le lignage ostéogénique sous l'action de différents facteurs, tels que des facteurs ostéo-inducteurs [22] (addition de BMP [bone morphogenetic protein]- 2 ou de BMP-7). Dans cette même optique, les matériaux peuvent être supplémentés avec ces protéines morphogénétiques avant leur implantation afin de stimuler la différenciation des CSM du tissu hôte. Les CSM peuvent être également orientées in situ par les propriétés mécaniques intrinsèques de la matrice $3 \mathrm{D}$ au sein de laquelle elles sont hébergées [1].

\section{Biomatériaux et contraintes mécaniques}

Parmi les biomatériaux les plus utilisés en ingénierie du tissu osseux, nous citerons les matériaux macroporeux à base de phosphate de calcium, les polymères synthétiques, naturels et/ou composites [24] qui donnent des résultats très prometteurs sur la différenciation des CSM, surtout dans des conditions dynamiques de culture [25].

$\varepsilon n$ effet, le tissu osseux étant un tissu continuellement soumis à plusieurs types et niveaux de sollicitations mécaniques, différents dispositifs comme les bioréacteurs sont développés pour mimer in vitro ces contraintes. Pendant de nombreuses années, seuls les ostéocytes étaient considérés comme des cellules mécanosensibles, mais la réponse des CSM à différents types de contraintes (compression, étirement, cisaillement) 


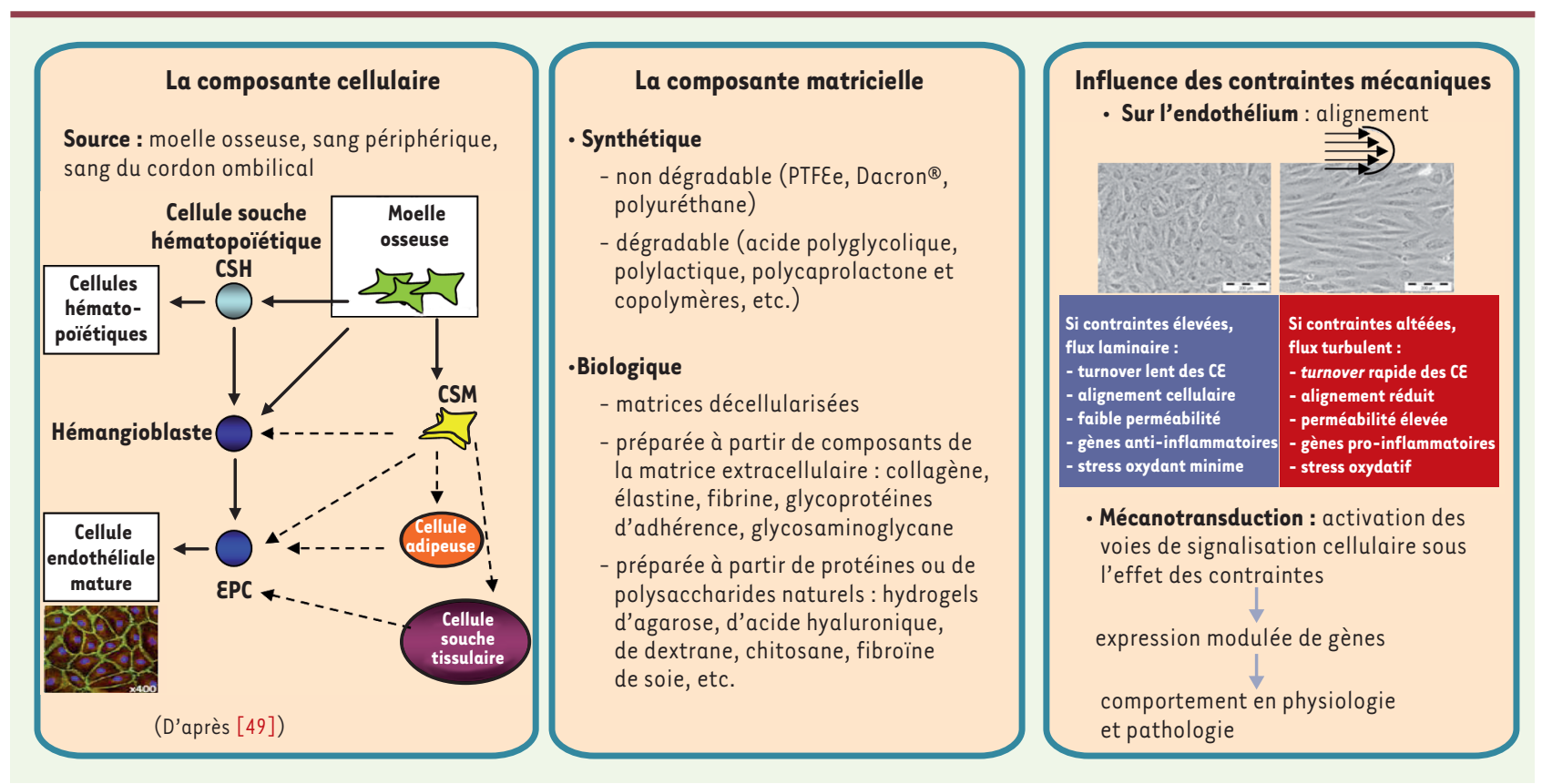

Figure 3. Ingénierie du tissu vasculaire : les cellules issues des CSH, des CSM, endothéliales et EPC, les biomatériaux et les contraintes mécaniques les mieux connues (contraintes de cisaillement). CSM : cellule souche mésenchymateuse ; CSH : cellules souches hématopoïétiques ; $\varepsilon P C$ : progéniteur épithélial circulant.

est à ce jour mieux identifiée. Les missions de ces bioréacteurs sont d'assurer la perfusion de ces matrices 3D, l'apport de nutriments aux CSM et l'élimination des déchets. Cette culture dynamique peut avoir des conséquences importantes sur la différenciation des CSM puis sur la formation osseuse et la vascularisation des tissus néoformés après implantation de ces matrices dans des modèles expérimentaux de régénération osseuse [26].

Le processus de vascularisation peut être également stimulé par l'introduction de progéniteurs endothéliaux associés aux CSM dans les matrices 3D [27]. De nombreux travaux décrivent l'intérêt des cocultures de progéniteurs du lignage endothélial et mésenchymateux pour favoriser un dialogue ostéoendothélial et stimuler in vivo à la fois l'ostéogenèse et l'angiogenèse des tissus néoformés [28]. Dans ce contexte, les interactions cellulaires jouent un rôle fondamental dans la différenciation des cellules souches, celles-ci formant le plus souvent des agrégats cellulaires en 3D [29].

Enfin, si les résultats obtenus avec ces nouveaux produits d'IT sont satisfaisants dans des modèles précliniques [30], le nombre d'essais cliniques reste encore trop limité [31]. Ils sont répertoriés pour un nombre faible de patients souffrant principalement de pertes massives de tissu osseux au niveau des os longs (tibia) [32] ou encore pour des applications en chirurgie maxillofaciale [33]. Les difficultés majeures rencontrées pour de tels essais cliniques sont liées aux aspects réglementaires de l'élaboration de ces produits de thérapie innovants (développement des produits d'IT en conditions GMP, good manufacturing practice) pour assurer une parfaite biosécurité pour le patient $[52](\rightarrow)$.

$(\rightarrow)$ Voir l'article de L. Sensebé et P. Bourin, page 297 de ce numéro

\section{Ingénierie tissulaire dans le domaine vasculaire : les cellules souches ont-elles un avenir?}

Les pathologies cardiovasculaires restent la principale cause de mortalité dans les pays occidentaux. Actuellement, face aux atteintes de l'intégrité du système vasculaire, des solutions existent : techniques curatives endovasculaires, techniques réparatrices de pontage du segment artériel défectueux par un substitut vasculaire. Ces substituts doivent répondre à une double exigence : combiner des propriétés structurales qui garantissent un comportement mécanique satisfaisant et des propriétés superficielles qui garantissent l'hémocompatibilité. Si la chirurgie de revascularisation appliquée aux artères de diamètre supérieur à $5-6 \mathrm{~mm}$ est performante à long terme avec les prothèses en Dacron ${ }^{\circledR}$ et en ePTFE (polytétrafluoroéthylène expansé) utilisées en clinique, aucun de ces matériaux ne convient pour remplacer des vaisseaux de plus petit diamètre. En effet, l'utilisation de substituts synthétiques présente deux limites majeures: ses propriétés (bio)mécaniques (faible compliance comparativement aux artères natives) et biologiques (non-recouvrement par un endothélium). Dans l'acception la plus étendue de l'IT appliquée au domaine vasculaire, plusieurs approches sont envisageables selon l'application visée: transplantation de cellules (confinées ou non) dans un territoire ischémié, équivalents de tissus dont la vascularisation doit être 
assurée/suscitée, fonctionnalisation de matrices ou de biomatériaux par des cellules in vitro ou par des revêtements de nature variable pour recruter in situ les cellules adéquates. Un des objectifs majeurs de ces constructions hybrides est en effet de présenter, à terme, au contact du sang, une surface cellulaire non thrombogène [51].

$\varepsilon n$ ingénierie vasculaire, des résultats cliniques probants sont rapportés avec des biomatériaux (dégradables ou non) associés - préalablement à leur implantation - à des cellules autologues dans la réalisation de pontages [34], de constructions vasculaires totalement autologues [35], ou dans la chirurgie des cardiopathies congénitales [36]. Selon les cas, les cellules ensemencées sont soit matures soit immatures et, dans ce cas, elles se différencieront in situ. Ainsi, les paramètres déterminants portent sur les choix d'un ou plusieurs types cellulaires et de leur source d'une part, et sur le choix des matériaux à coloniser d'autre part.

Cellules souches pluripotentes source de cellules endothéliales $\varepsilon n$ ce qui concerne la source cellulaire, les cellules souches offrent l'avantage théorique d'avoir un potentiel de prolifération élevé et d'être relativement accessibles pour l'ingénierie vasculaire. D'importants travaux ces dernières années contribuent à une meilleure connaissance des processus qui conduisent à la différenciation vasculaire: des cellules souches embryonnaires pluripotentes, placées dans des conditions de culture précises, ont la capacité de se différencier en cellules spécialisées correspondant à tous les tissus de l'organisme, y compris les tissus vasculaires [37]. Leur utilisation en thérapie vasculaire clinique [38] et l'ingénierie tissulaire permettraient de remédier aux limites rencontrées avec les cellules progénitrices endothéliales $(E P C)$ (voir ci-dessous). Des constructions tissulaires microvascularisées [38] qui utilisent ces cellules confinées dans des matrices 3D naturelles ou synthétiques (Figure 3) ont été ainsi développées.

D'autres cellules souches pluripotentes, les iPS, ont la capacité de se différencier en cellules de la paroi vasculaire (endothéliales [CE] et musculaires lisses [CML]) et d'en exprimer les marqueurs [39]. Des cellules souches résident aussi dans l'organisme adulte au sein de véritables entités spatiotemporelles dynamiques (niches) qui vont contrôler le destin de ces cellules dans les tissus, sous la dépendance de communications cellulaires [40].

\section{Utilisation des précurseurs endothéliaux}

La découverte des EPC en 1997 chez l'adulte a bousculé le dogme selon lequel la vasculogenèse serait restreinte à l'embryogenèse et a conduit à la mise en place de plusieurs essais cliniques sur le principe de l'induction d'une néovascularisation par l'implantation d'EPC dans le site ischémié [41]. Les résultats sont cependant controversés, qu'il s'agisse du mécanisme précis de l'amélioration de la perfusion vasculaire ou de celui du bénéfice clinique, s’il existe. En effet, les résultats de ces essais cliniques n'ont pas révélé le niveau d'efficacité que pouvaient laisser espérer les études précliniques. Un progéniteur endothélial EPC est un précurseur engagé dans la lignée endothéliale, mais en amont d'une CE mature [42]. Les EPC peuvent être isolés à partir de divers tissus : moelle osseuse, sang périphérique, paroi des vaisseaux sanguins, pulpe dentaire, foie, tissu adipeux, musculaire, nerveux, sang de cordon ombilical. Ils pourraient dériver à la fois des CSH mais aussi des CSM [42]. Un des avantages de l'utilisation des EPC à la place des cellules endothéliales matures pour endothélialiser des prothèses vasculaires est leur facilité de prélèvement puisqu'ils circulent dans le sang et résident dans des tissus facilement accessibles comme la moelle osseuse [43]. Mais pour Brown et al. [44], des critères doivent être remplis avant de pouvoir utiliser ces EPC ou les CE issues d'EPC pour l'ingénierie vasculaire. Il est possible de reconstituer in vitro un néoendothélium sur des substituts vasculaires synthétiques et biologiques à partir d'EPC issus de sang périphérique humain ou animal avant l'implantation en situation autologue [45].

\section{Autres sources de cellules endothéliales:}

\section{cellules souches mésenchymateuses}

Sur la base de preuves de concept obtenues chez l'animal, notamment chez le chien, Matsumura et al. [46] ont montré qu'il était non seulement réalisable mais efficace et utile chez l'homme d'ensemencer des matrices de polymère dégradables avec une suspension de cellules mononucléées de moelle osseuse autologue avant d'implanter ces produits d'ingénierie tissulaire. Leur remodelage in vivo aboutit en quelques mois à la maturation de véritables vaisseaux sanguins fonctionnels chez l'animal receveur: la présence de cellules musculaires lisses et de $C \varepsilon$ ayant été attribuée à la différenciation vasculaire à partir des cellules mononucléées. En réalité, des études récentes basées sur le même principe viennent d'invalider cette hypothèse en montrant chez la souris [47] que les matrices cellularisées se transforment en vaisseaux sanguins non par transdifférenciation des cellules mononucléées ou par leur incorporation dans de nouveaux vaisseaux, mais par l'intermédiaire d'une réaction inflammatoire de remodelage vasculaire.

Beaucoup d'études ont apporté la preuve que des cellules stromales issues du tissu adipeux contiennent une population de cellules souches adultes mésenchymateuses et d'EPC pouvant se différencier en plusieurs lignages comme les CML et les CE. Le tissu adipeux pourrait donc être une source cellulaire alternative séduisante et privilégiée. Des applications cliniques des cellules souches du tissu adipeux (ADSC) sont rapportées dans des essais cliniques de phases I et II dans des infarctus du myocarde ou des pathologies vasculaires périphériques. Ces essais montrent un potentiel thérapeutique évident des ADSC $[48,53](\rightarrow)$. $\rightarrow$ Voir l'article de V. Planat-Benard, page 280 de ce numéro 
Lorsque le revêtement endothélial ne peut pas être obtenu in vitro, certains auteurs l'envisagent in situ et développent des stratégies de fixation de divers agents proactifs (anticorps, aptamères, peptides, etc.). Les EPC circulantes pourraient ainsi être capturées à la surface de stents ou de prothèses fonctionnalisées puis se différencier [49]. Pour ce qui est du choix de biomatériaux candidats innovants à même de faciliter la croissance clonale de cellules souches humaines pluripotentes (embryonnaires ou iPS) tout en présentant des perspectives thérapeutiques réalistes, une élégante étude a récemment combiné, par une approche de relations structure-fonctions, les propriétés des matériaux et leurs performances biologiques pour la mise au point de substrats parfaitement définis en termes de mouillabilité, topographie et chimie de surface, module élastique de différents polymères [50].

\section{Conclusion}

Les récentes avancées de la biologie des cellules souches et des sciences des matériaux ont contribué à faire de l'ingénierie tissulaire une stratégie prometteuse pour la régénération des tissus squelettiques et vasculaires. Cependant, et malgré des résultats in vivo très encourageants, I'IT est encore loin de permettre la régénération d'un tissu totalement fonctionnel et comparable au tissu sain. Parallèlement, I'utilisation des CSM en médecine régénératrice reste encore limitée. $\varepsilon n$ effet, les capacités de régénération des CSM diminuent avec l'âge du donneur. L'amplification des CSM in vitro à long terme peut également entraîner l'apparition de modifications altérant leur phénotype. De plus, les données concernant les propriétés immunomodulatrices des CSM après différenciation sont contradictoires et devront être clarifiées avant leur possible utilisation clinique $[54](\rightarrow)$.

$\rightarrow$ Voir l'article de C. Ménard et K. Tarte, page 269 de ce numéro Finalement, rappelons que I'IT est une discipline encore jeune; le cadre réglementaire qui gère les produits d'IT n'était pas défini il y a trois ans. Néanmoins, les premiers produits issus de l'IT frappent aujourd'hui à la porte des essais cliniques, sachant qu'un règlement européen ( $C \varepsilon / 1394 / 2007)$ encadre depuis le 30 décembre 2008 le développement de ces produits de thérapie innovante (PTI) [52] $(\rightarrow)$.

\section{$(\rightarrow)$ Voir l'article de L. Sensebé et P. Bourin, page 297 de ce numéro}

\section{SUMMARY}

Stem cells for osteoarticular and vascular tissue engineering

Tissue damages or loss of organs often result in structural and metabolic changes that can cause serious complications. The therapeutic objective of tissue engineering $(T \varepsilon)$ is to recreate, regenerate or restore function of damaged tissue. $T \varepsilon$ is based on the coalescence of three components: a scaffold or matrix from natural or synthetic origin biodegradable or not, reparative cells and signals (hypoxia, mechanical stress, morphogens...). Articular cartilage, bone and blood vessels are tissues for which $T \varepsilon$ has progressed significantly, from basic research to clinical trials. If biomaterials must exhibit different properties depending on the tissue to regenerate, the cellular component of $T \varepsilon$ is mostly represented by stem cells notably adult mesenchymal stem cells harvested from bone marrow or adipose tissue. In recent years, progress has been made in our understanding of the biological mechanisms that govern stem cell differentiation and in the development of materials with controlled physicochemical and biological properties. However, many technological barriers and regulations concerns have to be overcome before tissue engineering enters into the therapeutic arsenal of regenerative medicine. This review aims at highlighting the progress in the use of stem cells for engineering osteoarticular and vascular tissues. $\diamond$

\section{CONFLIT D'INTÉRÊTS}

C. Vinatier déclare avoir des liens permanents avec l'entreprise Graftys SA. L. Bordenave, J. Guicheux et J. Amédée déclarent n 'avoir aucun conflit d'intérêts concernant les données publiées dans cet article.

\section{RÉFÉRENCES}

1. Engler AJ, Sen S, Sweeney HL, Discher DE. Matrix elasticity directs stem cell lineage specification. Cell $2006 ; 126: 677-89$.

2. Kassem M, Abdallah BM. Human bone-marrow-derived mesenchymal stem cells: biological characteristics and potential role in therapy of degenerative diseases. Cell Tissue Res 2008 ; 331 : 157-63.

3. Gomillion CT, Burg KJ. Stem cells and adipose tissue engineering. Biomaterials $2006 ; 27: 6052-63$.

4. Johnstone B, Hering TM, Caplan Al, et al. In vitro chondrogenesis of bone marrow-derived mesenchymal progenitor cells. Exp Cell Res 1998 ; $238: 265-72$.

5. Koga H, Muneta T, Nagase T, et al. Comparison of mesenchymal tissuesderived stem cells for in vivo chondrogenesis: suitable conditions for cell therapy of cartilage defects in rabbit. Cell Tissue Res $2008 ; 333: 207-15$

6. Scotti C, Tonnarelli B, Papadimitropoulos A, et al. Recapitulation of endochondral bone formation using human adult mesenchymal stem cells as a paradigm for developmental engineering. Proc Natl Acad Sci USA 2010 ; $107: 7251-6$

7. Vinatier C, Mrugala D, Jorgensen C, et al. Cartilage engineering: a crucial combination of cells, biomaterials and biofactors. Trends Biotechnol 2009 ; 27 : 307-14.

8. Merceron C, Vinatier C, Portron S, et al. Differential effects of hypoxia on osteochondrogenic potential of human adipose-derived stem cells. AmJ Physiol Cell Physiol $2010 ; 298$ : C355-64

9. Terraciano V, Hwang N, Moroni L, et al. Differential response of adult and embryonic mesenchymal progenitor cells to mechanical compression in hydrogels. Stem Cells $2007 ; 25: 2730-8$.

10. Pelttari $K$, Winter A, Steck $\varepsilon$, et al. Premature induction of hypertrophy during in vitro chondrogenesis of human mesenchymal stem cells correlates with calcification and vascular invasion after ectopic transplantation in SCID mice. Arthritis Rheum 2006 ; 54 : 3254-66.

11. Giovannini S, Diaz-Romero J, Aigner T, et al. Micromass co-culture of human articular chondrocytes and human bone marrow mesenchymal stem cells to investigate stable neocartilage tissue formation in vitro. Eur Cell Mater $2010 ; 20: 245-59$.

12. Merceron C, Portron S, Masson M, et al. Cartilage tissue engineering: from hydrogel to mesenchymal stem cells. Biomed Mater Eng $2010 ; 20: 159-66$.

13. Wakitani S, Imoto K, Yamamoto T, et al. Human autologous culture expanded bone marrow mesenchymal cell transplantation for repair of cartilage defects in osteoarthritic knees. Osteoarthritis Cartilage 2002 ; 10 : 199-206.

14. Wakitani S, Mitsuoka T, Nakamura N, et al. Autologous bone marrow stromal cell transplantation for repair of full-thickness articular cartilage defects in human patellae: two case reports. Cell Transplant $2004 ; 13$ : 595-600.

15. Wakitani S, Nawata M, Tensho K, et al. Repair of articular cartilage defects in the patello-femoral joint with autologous bone marrow mesenchymal cell transplantation: three case reports involving nine defects in five knees. J Tissue Eng Regen Med 2007 ; 1 : 74-9. 


\section{RÉFÉRENCES}

16. Dawson $\varepsilon$, Mapili G, Erickson K, et al. Biomaterials for stem cell differentiation. Adv Drug Deliv Rev $2008 ; 60: 215-28$

17. Friedenstein AJ, Chailakhyan RK, Gerasimov UV. Bone marrow osteogenic stem cells: in vitro cultivation and transplantation in diffusion chambers. Cell Tissue Kinet 1987 ; $20: 263-72$.

18. Bueno EM, Glowacki J. Cell-free and cell-based approaches for bone regeneration. Nat Rev Rheumatol $2009 ; 5: 685-97$.

19. Xiao Y, Qian H, Young WG, Bartold PM. Tissue engineering for bone regeneration using differentiated alveolar bone cells in collagen scaffolds. Tissue Eng $2003 ; 9$ : 1167-77.

20. Jukes JM, van Blitterswijk CA, de Boer J. Skeletal tissue engineering using embryonic stem cells. J Tissue Eng Regen Med 2008; 4 : 165-80.

21. Li F, Bronson S, Niyibizi C. Derivation of murine induced pluripotent stem cells (iPS) and assessment of their differentiation toward osteogenic lineage. J Cell Biochem $2010 ; 109$ : 643-52

22. Reddi AH. Morphogenesis and tissue engineering of bone and cartilage: inductive signals, stem cells, and biomimetic biomaterials. Tissue Eng $2000 ; 6: 351-9$.

23. Marie P. Différenciation, fonction et contrôle de l'ostéoblaste. Med Sci (Paris) 2001 ; 17 : 1252-9.

24. Swetha M, Sahithi K, Moorthi A, et al. Biocomposites containing natural polymers and hydroxyapatite for bone tissue engineering. Int J Biol Macromol 2011 (sous presse).

25. Datta N, Pham QP, Sharma U, et al. In vitro generated extracellular matrix and fluid shear stress synergistically enhance 3D osteoblastic differentiation. Proc Natl Acad Sci USA 2006; $103: 2488-93$.

26. Scherberich A, Galli R, Jaquiery C, et al. Three-dimensional perfusion culture of human adipose tissue-derived endothelial and osteoblastic progenitors generates osteogenic constructs with intrinsic vascularization capacity. Stem Cells $2007 ; 25$ : 1823-9.

27. Yu H, VandeVord PJ, Mao L, et al. Improved tissue-engineered bone regeneration by endothelial cell mediated vascularization. Biomaterials 2009 ; $30: 508-17$.

28. Grellier M, Bordenave L, Amédée J. Cell-to-cell communication between osteogenic and endothelial lineages: implications for tissue engineering. Trends Biotechnol $2009 ; 27$ : 562-71.

29. Chen SS, Fitzgerald W, Zimmerberg J, et al. Cell-cell and cell-extracellular matrix interactions regulate embryonic stem cell differentiation. Stem Cells $2007 ; 25: 553-61$.

30. Reichert JC, Saifzadeh S, Wullschleger ME, et al. The challenge of establishing preclinical models for segmental bone defect research. Biomaterials $2009 ; 30: 2149-63$.

31. Chatterjea A, Meijer GJ, van Blitterswijk C, de Boer J. Clinical applications of human mesenchyma stromal cells for bone tissue engineering. Stem Cell Int 2011 (sous presse).

32. Quarto R, Mastrogiacomo M, Cancedda R, et al. Repair of large bone defects with the use of autologous bone marrow stromal cells. $N$ Engl J Med 2001 ; 344 : 385-6.

33. Meijer GJ, de Bruijn JD, Koole R, et al. Cell based bone tissue engineering in jaw defects. Biomaterials 2008 ; 29 : 3053-61

34. Deutsch M, Meinhart J, Zilla $\mathrm{P}$, et al. Long-term experience in autologous in vitro endothelialization of infrainguinal ePTFE grafts. J Vasc Surg 2009; 49 : 352-62.

35. McAllister TN, Maruszewski M, Garrido SA, et al. Effectiveness of haemodialysis access with an autologous tissue-engineered vascular graft: a multicentre cohort study. Lancet 2009 ; $373: 1440-6$.

36. Shin'oka T, Matsumura G, Hibino N, et al. Midterm clinical result of tissue-engineered vascular autografts seeded with autologous bone marrow cells. J Thorac Cardiovasc Surg 2005 ; $129: 1330-8$

37. Hanjaya-Putra D, Gerecht S. Vascular engineering using human embryonic stem cells. Biotechnol Prog $2009 ; 25: 2-9$.

38. Yamahara K, Itoh H. Potential use of endothelial progenitor cells for regeneration of the vasculature. Ther Adv Cardiovasc Dis $2010 ; 3: 17-27$

39. Taura D, Sone DM, Homma K, et al. Induction and isolation of vascular cells from human induced pluripotent stem cells-brief report. Arterioscler Thromb Vasc Biol 2009; 29 : 1100-3.
40. Lataillade JJ, Brunet de la Grange P, Uzan G, Le Bousse-Kerdilès MC. Les cellules souches ont-elles l'âge de leur niche? À la recherche d'un sérum de jouvence. Med Sci (Paris) 2010 ; 27 : ???-???.

41. Krenning G, van Luyn MJ, Harmsen MC. Endothelial progenitor cell-based neovascularization: implications for therapy. Trends Mol Med 2009 ; $15: 180-9$.

42. Smadja DM, Cornet A, Emmerich J, et al. Endothelial progenitor cells: characterization, in vitro expansion, and prospects for autologous cell therapy. Cell Biol Toxicol $2007 ; 23: 223-39$.

43. Ward MR, Stewart DJ, Kutryk MJ. Endothelial progenitor cell therapy for the treatment of coronary disease, acute $\mathrm{MI}$, and pulmonary arterial hypertension: current perspectives. Catheter Cardiovasc Interv 2007 ; $70: 983-98$.

44. Brown MA, Wallace CS, Angelos M, Truskey GA. Characterization of umbilical cord blood derived late outgrowth endothelial progenitor cells exposed to laminar shear stress: Tissue Eng Part A 2009 ; 15 : 3575-87.

45. Kaushal S, Amiel GE, Guleserian KJ, et al. Functional small-diameter neovessels created using endothelial progenitor cells expanded ex vivo. Nat Med $2001 ; 7: 1035-40$

46. Matsumura G, Hibino N, Ikada Y, et al. Successful application of tissue engineered vascular autografts: clinical experience. Biomaterials 2003; $24: 2303-8$.

47. Roh JD, Sawh-Martinez R, Brennan MP, et al. Tissue-engineered vascular grafts transform into mature blood vessels via an inflammation-mediated process of vascular remodeling. Proc Natl Acad Sci USA $2010 ; 107: 4669-74$.

48. Hong SJ, Traktuev DO, March KL. Therapeutic potential of adipose-derived stem cells in vascular growth and tissue repair. Curr Opin Organ Transplant $2010 ; 15: 86-91$.

49. Avci-Adali M, Ziemer G, Wendel HP. Induction of EPC-homing on biofunctionalized vascular grafts for rapid in vivo self-endothelialization. A review of current strategies. Biotech Adv $2010 ; 28$ : 119-29.

50. Mei Y, Saha K, Bogatyrev SR, et al. Combinatorial development of biomaterials for clonal growth of human pluripotent stem cells. Nat Mater $2010 ; 9: 768-78$.

51. Meddahi-Pellé A, Bataille I, Subra P, Letourneur D. Biomatériaux vasculaires : du génie biologique et médical au génie tissulaire. Med Sci (Paris) $2004 ; 20: 679-84$

52. Sensebé $L$, Bourin P.Cellules souches mésenchymateuses : production a usage clinique et contraintes sécuritaires. Med Sci (Paris) 2011 ; 27 : 297 302.

53. Jorgensen C, Deschaseaux F, Planat-Benard V, Gabison E. Les cellules souches mésenchymateuses : actualités thérapeutiques. Med Sci (Paris) $2011 ; 27: 275-84$

54. Ménard C, Tarte K. Immunosuppression et cellules souches mésenchymateuses : mieux comprendre une propriété thérapeutique majeure. Med Sci (Paris) $2011 ; 27: 269-74$

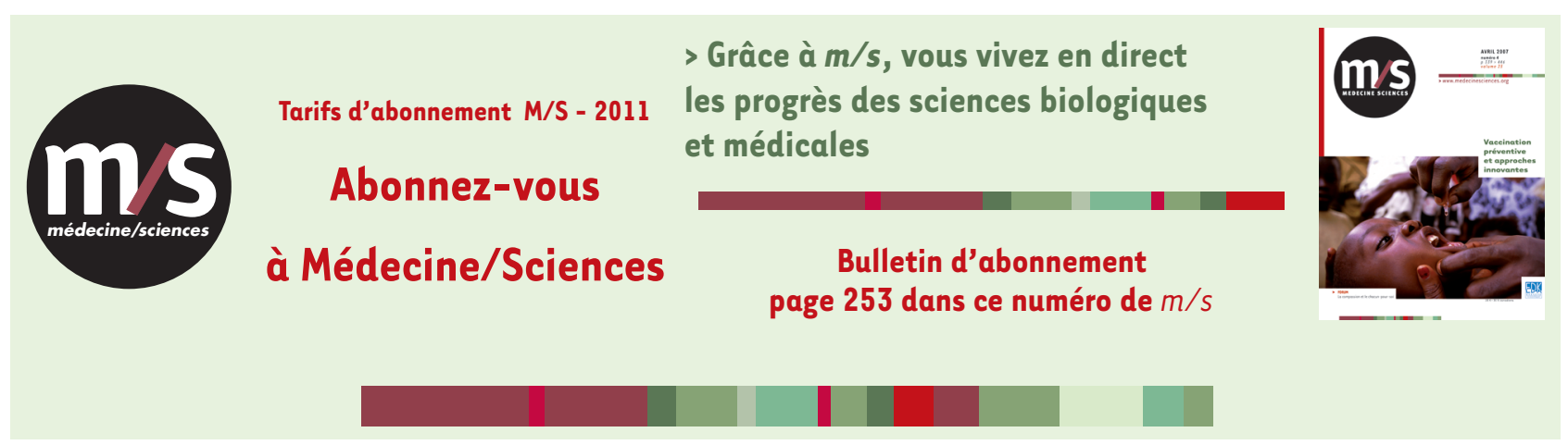

TIRÉS À PART

J. Amédée 